\section{P-152 IMPROVING QUALITY AND PATIENT EXPERIENCE BY LEARNING FROM DEATHS REVIEWS - THE HOSPICE WAY}

Tracey Grint, Sarah Onions. St Richard's Hospice, Worcester, UK

10.1136/spcare-2021-Hospice. 168

Background Over recent decades multiple health care inquiries including Shipman; Mazars; Francis; and Gosport have identified failings in care (Norris \& Shepheard, 2017) providing important recommendations to help create a health and care system that can identify failings in patient care before irreversible harm or avoidable deaths occur. Additional research has suggested that quality gaps can also be identified though mortality review (Kobewka, van Walraven, Turnbull, et al., 2017). In June 2019 our commissioners asked our hospice to perform mortality reviews on $5 \%$ of our deaths.

Aims The quality leads wanted to create a hospice specific learning from deaths (LFD) tool to support both random review and specific deeper dives into hospice deaths.

To take a wider learning and development approach to improve quality of our care and patient experience in a robust and time efficient way.

Methods We examined mortality review models including the National Mortality Case Record Review Programme's Structure judgment review and the Gold Standards Framework significant event analysis to create our inpatient hospice specific LFD tool. This tool helps identify near miss events and recognise areas of improvement that do not reach the threshold for triggering our incident reporting process. The review group, led by our Care Quality Lead, includes senior nursing and medical input and an external medical reviewer.

Results Two patients are reviewed monthly, identified randomly by an administrator; by multidisciplinary team request, following a sudden or unexpected death; or as a result of patient and family feedback. An action tracker is reviewed monthly and an annual review identifies themes or wider actions.

Outcome This process has become a key trigger for quality improvement in the hospice. It also prompted identification of a direct pathway to request hospital mortality review. It has enhanced our open culture for learning and augmented service development to improve the quality of care and patient and family experience.

\section{P-153 RETROSPECTIVE ANALYSIS OF CASE NOTES REVIEWING UNEXPECTED DEATHS ON A SPECIALIST PALLIATIVE CARE UNIT}

Gabriella Swanepoel, Emma Longford. Wirral Hospice St Johns, Liverpool, UK

10.1136/spcare-2021-Hospice.169

Retrospective analysis of case notes to review the unexpected deaths on a specialist palliative care unit. Our aim was to review and analyse patient notes from our inpatient specialist palliative care unit where death had not been recognised by the treating team.

We analysed twenty sets of case notes from March 2020 to March 2021. The following questions were answered; length of admission, documentation about expectation of death and discussion with the patient and relatives about this, last recorded phase of illness and functional status in MDT discussions and if active treatment was being given at the time of death. A thematic analysis was undertaken of these results and several trends were evident. We found that patients with a shorter admission and younger patients who have been resistant to advance care planning discussions were less likely to be identified for the end-of-life record of care.

From this research we have identified the need for more open discussions around expectations of death, we have increased the number of professionals undertaking advance care planning courses including doctors and nurses on the inpatient unit. We acknowledge that deaths cannot always be expected, however, we would like to implement more robust advance care planning discussions, proactive management and maximum exposure to MDT management for all patients.

\section{P-154 NO SURPRISES? REDUCING RISK THROUGH EFFECTIVE CONTRACT MANAGEMENT}

Kevin McGill, Laura Brisley. St Helena Hospice, Colchester, UK

10.1136/spcare-2021-Hospice. 170

Background Effective contract management drives value for money and delivers successful contractual outcomes (Cabinet Office, The Sourcing playbook: Government guidance on service delivery, including outsourcing, insourcing, mixed economy sourcing and contracting, 2021). If not carefully managed, notice periods are missed, but resourcing operations is a challenge (Copeman et al., 2017), and reactive management is costly. Contract maintenance is therefore critical to the success of contract administration (Chartered Institute of Procurement \& Supply, 2013).

Aim To create a bespoke data management solution to collate and manage any data efficiently and create a central platform for managing contracts effectively, and reduce the financial and operational risks presented by poorly managed contracts, and to spread this best practice across the organisation.

Method We identified a risk in that contracts can carry extensive complexity and financial risk. We held a meeting and agreed to create a Contract Register module and collate key contractual information and copy of contract. A template was prepared for recording extant contracts, and the Contract Register was populated, setting trigger fields to give notice period warnings to named contract owners, and agreed on best practice regular review triggers for higher risk contracts, with an activity $\log$ facility to document any changes or meetings with the contractor or raised issues with performance delivery.

Results The module is currently in use for the Estates team, and now flags expiring contracts in a timely manner allowing a sufficient time period to conduct an effective contract review or to initiate a procurement exercise if so required. This will be expanded across all other departments for full contractual visibility for all services and closer management of financial and operational risks of respective contractual commitments.

Conclusion Contracts can be complex to manage and financially cumbersome when overlooked (Carter, Kirby, Jackson, 2014). Our contracts are now risk managed pro-actively through our bespoke Contract Register, which provides reports on contracts due for renewal or review. Going forward, we intend to share this learning and platform with other departments and hospices. 